\title{
PROPER KNOT THEORY IN OPEN 3-MANIFOLDS
}

\author{
PETER CHURCHARD AND DAVID SPRING
}

\begin{abstract}
This paper introduces a theory of proper knots, i.e., smooth proper embeddings of $\mathbf{R}^{1}$ into open 3-manifolds. Proper knot theory is distinguished by the fact that proper isotopies of knots are not ambient in general. A uniqueness theorem for proper knots is proved in case the target manifold is the interior of a one-dimensional handlebody.
\end{abstract}

1. Introduction. In this paper, we consider the theory of smooth, proper embeddings of $\mathbf{R}^{1}$ into open 3-manifolds, hereafter referred to as the theory of proper knots. Two proper knots are equivalent, or isotopic, if they are connected by a smooth, proper isotopy of embeddings of $\mathbf{R}^{1}$ into the target open 3-manifold. This notion of equivalence leads to a nonambient classification theory of proper knots: In general, an equivalence of proper knots cannot be covered by a smooth isotopy of diffeomorphisms of the ambient open 3-manifold.

To illustrate proper knot theory, we consider the cases of the following open 3-manifolds:

(a) $\mathbf{R}^{3}$,

(b) $S^{1} \times \mathbf{R}^{2}$ (the interior of the solid torus),

(c) $S^{2} \times \mathbf{R}$,

(d) $\operatorname{int}\left(D^{2} \times D^{1} \cup_{h} S^{2} \times D^{1}\right)$, the interior of the smooth, compact 3-manifold obtained by adding a 1-handle to a thickened $S^{2}$ (i.e., a punctured $S^{2} \times S^{1}=$ $\left.S^{2} \times S^{1}-p\right)$.

Topologists have known for some time that there is only one equivalence class of proper knots in $\mathbf{R}^{3}$. Proposition A below proves this result (which does not seem to appear in the literature) in the slightly more general context of smooth, proper embeddings of $\mathbf{R}^{n}$ into $\mathbf{R}^{s}, s \geq n+1$. Our viewpoint is that Proposition A shows that a proper knot in $\mathbf{R}^{3}$ may be "combed out" along a radial vector field in $\mathbf{R}^{3}$, leaving the inclusion map $i: \mathbf{R}^{1} \rightarrow \mathbf{R}^{3}$. The idea of combing out a proper knot along a suitable vector field in a manifold is made more precise in $\S 2$ and constitutes the main geometrical construction of the paper.

Our main result, Theorem A, is that proper knots may be combed out in certain smooth, open 3-manifolds. These are obtained as the interior of a (possibly noncompact) 3-manifold which is formed by adding a sequence of 1-handles to a 0 -handle. These manifolds have one end. Consequently, in such a 3-manifold, there is a unique proper knot up to equivalence. In particular, up to equivalence, there is a unique proper knot in manifold (b) above despite the fact that (b) is not simply connected.

Received by the editors December 27, 1986.

1980 Mathematics Subject Classification (1985 Revision). Primary 57M30. 
Algebraic invariants. In order to obtain computable algebraic invariants, we consider the special case of proper knots in int $M$, the interior of a compact, connected, $C^{\infty} 3$-manifold $M$ with nonempty, connected boundary $\partial M$. Since $M$ is compact, the one-point compactification of int $M$ is homeomorphic to $X=M \cup C \partial M$, the space obtained from $M$ by adjoining a cone along $\partial M$. A proper knot in int $M$ defines a closed loop $\alpha:([0,1],\{0,1\}) \rightarrow(X, *)(*$ is the cone point) such that $\alpha^{-1}(*)=\{0,1\}$. An isotopy class of proper knots defines an element of $\pi_{1}(X, *)$. This shows that there is a map,

$$
\Phi: \operatorname{Emb}\left(\mathbf{R}^{1}, \text { int } M\right) \rightarrow \pi_{1}(X, *)
$$

where $\operatorname{Emb}\left(\mathbf{R}^{1}\right.$, int $\left.M\right)$ denotes the set of isotopy classes of proper knots in int $M$. Since $X$ is triangulable as a finite complex, one easily verifies that $\Phi$ is onto. We further remark that if $\partial M=S^{2}$, then $\pi_{1}(X, *)=\pi_{1}(M)$.

In case $M=D^{3}$ or the solid $n$-hole torus (or the solid $n$-hole Klein bottle), then $\pi_{1}(X, *)=0$. For these special manifolds, Theorem $\mathrm{A}$ implies that the map $\Phi$ is injective. That is, up to isotopy, there is only one proper $\operatorname{knot}$ in int $M$.

To demonstrate proper knot invariants, then because of Theorem $\mathrm{A}$, we will examine examples with 2-handles or more than one end. Manifold (d) above has one 2-handle and one end. Manifold (c) has two ends. We have some rudimentary information about the classification of proper knots in the presence of 2-handles. To illustrate this, we consider three proper knots in the manifold (d) and they are the proper arcs $A B, C D$ and the proper knot $K_{m}$ which is contained in the open submanifold $S^{1} \times \mathbf{R}^{2}$ of manifold (d) and winds around $m$-times, $m \in \mathbf{Z}$. In fact $K_{0} \equiv A B$ and $K_{1} \equiv C D$.

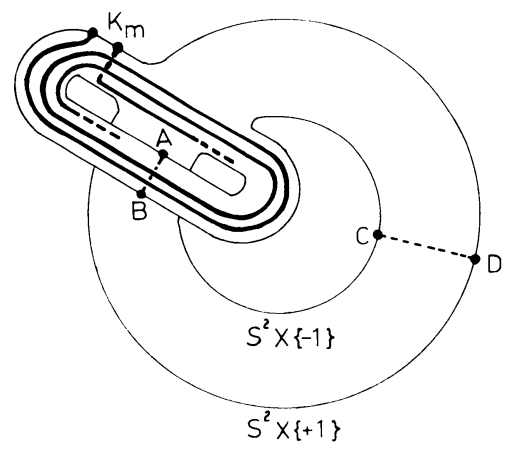

FIGURE 1

The proper knots $A B, C D$ and $K_{m}(m>1)$ are not equivalent since their intersection numbers with the compact 2-cycle $S^{2} \times\{0\}\left(\subset S^{2} \times D^{1}\right)$ are 0,1 and $m$ respectively. Note that the collection of knots, $K_{m}$, realize the invariant $\pi_{1}(X, *) \cong \mathbf{Z}$ in this case.

However, as a consequence of Theorem $\mathrm{A}, K_{m}$ and $A B$ are equivalent when viewed as proper knots in $S^{\mathbf{1}} \times \mathbf{R}^{2}$.

When more than one end is involved, we may define intersection and linking numbers between proper knots which are invariant under proper isotopy. In Figure 2 (a), we consider the manifold formed by removing four points from $\mathbf{R}^{2}$. The 


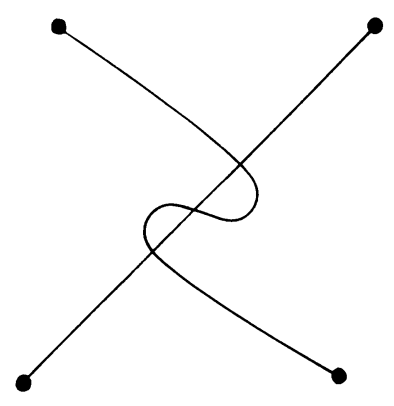

(a)

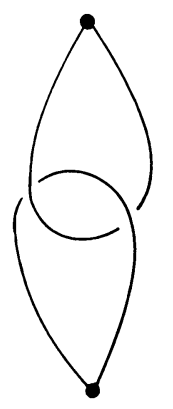

(b)

\section{FIGURE 2}

two proper knots that run between its four ends meet transversely to give a welldefined intersection number. In Figure 2(b), we similarly consider $\mathbf{R}^{3}$ minus two points and observe that the two proper knots depicted there have a well-defined linking number. The consideration of these examples generalizes to an intersection theory in open manifolds with multiple ends for cycles with closed supports.

These sorts of invariants, together with covering space theory, can be used to analyze proper knots. For example, in Figure 3(a), the proper knot $K$ in manifold (d) has intersection number 0 with $S^{2} \times\{0\}$, but it is not equivalent to the trivial arc $A B$. This can be seen as follows: since manifold (d) is $S^{2} \times S^{1}-p$, then $K$ lifts to the infinite chain $K^{\prime}$ in the universal cover $S^{2} \times \mathbf{R}-\mathbf{Z}$. $K$ is not equivalent to $A B$ since adjacent links in $K^{\prime}$ have linking number \pm 1 . This example also shows that properly homotopic knots need not be properly isotopic.

Turning now to the 3-manifold (c), a proper knot in $S^{2} \times \mathbf{R}$ which meets $S^{2} \times\{0\}$ transversely in exactly one point is equivalent, via combing out, to the proper arc $\{q\} \times \mathbf{R}, q \in S^{2}$, which has an appropriate orientation selected from the two possible ones. Because (c) has two ends, it is easy to see that there are at least four mutually inequivalent proper knots in $S^{2} \times \mathbf{R}$. In addition, the knot combing technique shows that there is a unique equivalence class of proper knots which send both ends of $\mathbf{R}$ to a given end of $S^{2} \times \mathbf{R}$. By contrast, the case where the ends of $\mathbf{R}$ are sent to

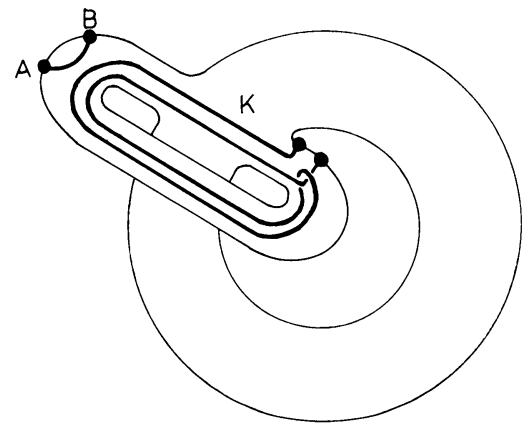

(a)

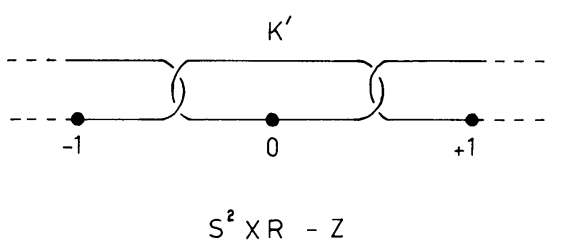

(b)

FIGURE 3 
distinct ends of $S^{2} \times \mathbf{R}$ seems, at first sight, to be much more complex. Here, the above transversality condition is not satisfied by many of these proper knots with respect to any essential, smoothly embedded $S^{2}$ in $S^{2} \times \mathbf{R}$. The wild arc (due to Fox [2]) which is shown in Figure 4(iv) is an interesting example of this. Figure 4(i)-(iv) indicate how it is actually equivalent to the trivial arc. We see that (ii) is equivalent to (i) via combing out, i.e., we push all the knotted behaviour to $-\infty$. We may then "knit" another loop in (ii) by pulling $B$ through $D$, hence showing that (ii) is equivalent to (iii). By continuing this knitting process out to $\infty$, we finally end up at (iv) via a proper isotopy. Notice that this is a local construction. In fact, ignoring orientation, all proper knots that run between the two ends of $S^{2} \times \mathbf{R}$ are equivalent by proper isotopies, as will be shown in a forthcoming paper.

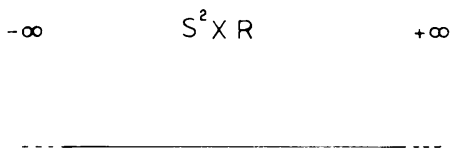

(i)

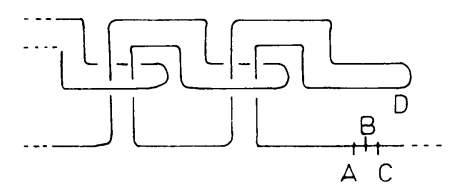

(ii)

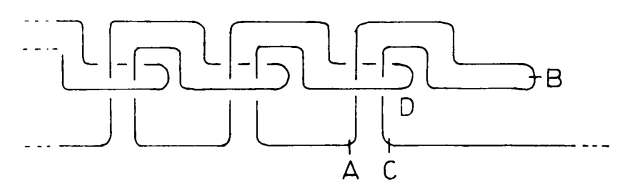

(iii)

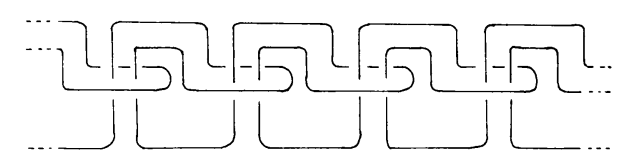

(iv)

FIGURE 4

In $\S 2$, we close with a generalisation of Theorem A to the case of smooth, proper embeddings of $\mathbf{R}^{p}$ into certain smooth, open $r$-manifolds, $r \geq p+1$.

The study of examples of proper knots in open 3-manifolds indicates that in each equivalence class of proper embeddings, there should be "locally trivial" proper knots: the locally knotted behaviour in any 3-ball has been combed out to infinity. In $\S 3$, we conjecture the existence of locally trivial representatives in any isotopy class of proper knots.

2. Definitions and theorems. A continuous map, $f: X \rightarrow Y$, is proper if $f^{-1}(K) \subset X$ is compact for each compact $K \subset Y$. In case $X$ is compact, any continuous map is proper. In what follows, we work in the category of smooth, open manifolds and smooth, proper maps. Thus, in this category, a homotopy is a proper map. An isotopy of embeddings is similarly a proper map and hence note that each stage of the isotopy is a proper embedding. Earlier results in this category were obtained by D. Spring [5], G. Bourgeois and D. Spring [1].

Throughout this paper, $B^{n}(\varepsilon)$ denotes the closed ball, radius $\varepsilon$, about the origin $0 \in \mathbf{R}^{n}$.

PROPOSITION A. Let $f: \mathbf{R}^{n} \rightarrow \mathbf{R}^{s}, s \geq n+1$, be any smooth map.

(a) If $f$ is proper, then there is a smooth, proper homotopy connecting $f$ to the inclusion $\iota: \mathbf{R}^{n} \rightarrow \mathbf{R}^{s}$. 
(b) If $f$ is a proper embedding, then the homotopy in part (a) may be taken to be a smooth, proper isotopy of embeddings to the inclusion $\iota: \mathbf{R}^{n} \rightarrow \mathbf{R}^{s}$.

PROOF. As it is more germane to this paper, we will only prove part (b), noting that part (a) follows in a similar fashion. So suppose $f: \mathbf{R}^{n} \rightarrow \mathbf{R}^{s}$ is a smooth, proper embedding. Using the fact that any two smoothly embedded $n$ discs are smoothly isotopic in $\mathbf{R}^{s}$ and then applying the isotopy extension theorem, we may assume that for some $\varepsilon>0, f$ is the inclusion on $B^{n}(\varepsilon)$ into $B^{s}(\varepsilon)$ and that $f\left(\mathbf{R}^{n}-B^{n}(\varepsilon)\right) \subset \mathbf{R}^{s}-B^{s}(\varepsilon)$. The required isotopy is then given by $F: \mathbf{R}^{n} \times[0,1] \rightarrow$ $\mathbf{R}^{s}$ where

$$
\begin{array}{ll}
(x, \mu) \rightarrow 1 / \mu \cdot f(\mu x) & \text { if } \mu>0, \\
(x, \mu) \rightarrow(x, 0)=\iota(x) & \text { if } \mu=0,
\end{array}
$$

Notice that $\forall \mu>0$, the $\mu$ th stage of $F, F_{\mu}=F(\cdot, \mu)$, agrees with $\iota$ on $B^{n}(\varepsilon / \mu)$ and that $\varepsilon / \mu \rightarrow \infty$ as $\mu \rightarrow 0$. This shows that $F$ is smooth on $\mathbf{R}^{n} \times\{0\}$. $F$ is clearly smooth on $\mathbf{R}^{n} \times(0,1]$. So as $\mu \rightarrow 0$, we are "combing out the knots in $f$ " and leaving behind the inclusion $\iota$.

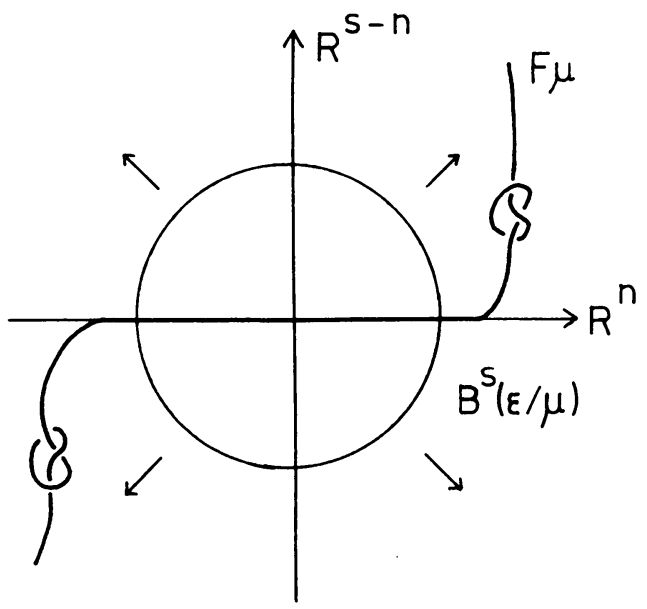

FIGURE 5

For any $W \subset \mathbf{R}^{n} \times[0,1]$, let $w d W$, the width of $W$, be defined by

$$
\operatorname{wd} W=\sup \{\|x\| \mid(x, y) \in W\} \text {. }
$$

To show that $F$ is proper, it suffices to prove that given any compact $K \subset \mathbf{R}^{s}$, then $\operatorname{wd}\left(F^{-1}(K)\right)<\infty$. Let $K \subset B^{s}\left(r_{0}\right), r_{0}>0$. Then $\exists \mu_{0}>0$ such that $F_{\mu_{0}}(x)=\iota(x)$ $\forall x \in B^{n}\left(r_{0}\right)$, so $\forall 0 \leq \mu \leq \mu_{0}, K \cap F_{\mu}(\mathbf{R})=K \cap \iota\left(B^{n}\left(r_{0}\right)\right)$. Hence

$$
\begin{aligned}
F^{-1}(K) & =\bigcup_{0 \leq \mu \leq 1} F_{\mu}^{-1}(K) \times\{\mu\} \\
& =\left(\iota^{-1}(K) \cap B^{n}\left(r_{0}\right) \times\left[0, \mu_{0}\right]\right) \cup \bigcup_{0<\mu_{0} \leq \mu \leq 1} F_{\mu}^{-1}(K) .
\end{aligned}
$$


Now if $\mu>0$, then $F_{\mu}^{-1}(K)=\mu f^{-1}(1 / \mu \cdot K)$ and for $\mu \geq \mu_{0}$, we have $1 / \mu \cdot K \subset$ $1 / \mu_{0} \cdot K \subset 1 / \mu_{0} \cdot B^{s}\left(r_{0}\right)$. So,

$$
\mathrm{wd}\left(\bigcup_{\mu_{0} \leq \mu \leq 1} F_{\mu}^{-1}(K) \times\{\mu\}\right) \leq \mathrm{wd}\left(f^{-1}\left(\frac{1}{\mu_{0}} \cdot B^{s}\left(r_{0}\right)\right) \times\left[\mu_{0}, 1\right]\right)<\infty
$$

(since $f$ is proper).

Hence $\operatorname{wd}\left(F^{-1}(K)\right)<\infty$.

The above may be extended to more general target manifolds other than $\mathbf{R}^{p}$ when we look at the "combing out" procedure in terms of vector fields. Consider the special case $f: \mathbf{R}^{1} \rightarrow \mathbf{R}^{3}$ and the radial vector field $V$ on $\mathbf{R}^{3}$ given by $V(p)=p$, $p \in \mathbf{R}^{3}$. To show the above proposition, we could have isotoped $f$ near the origin in $\mathbf{R}^{1}$ to lie along the paths of two trajectories of $V$ emanating from $0 \in \mathbf{R}^{3}$ (e.g., the $x$-axis). Then using the flow of $V, f$ could be "combed out" along these trajectories. In this fashion, all the "knotted" behaviour of $f$ is swept out to $\infty$ by a proper isotopy, leaving behind the inclusion $\mathbf{R}^{1} \rightarrow \mathbf{R}^{3}$. These ideas lead to the following theorem.

THEOREM A. Let $M$ be an open, connected, smooth 3-manifold which admits a smooth, proper Morse function $m: M \rightarrow \mathbf{R}$ such that

(i) $m(M)=[0, \infty)$,

(ii) the critical points of $m$ have index 0 or 1 only.

Then, up to smooth, proper isotopy, there is a unique, smooth, proper embedding $g: \mathbf{R} \rightarrow M$.

PROOF. We first note that the set of critical points of $m$ is at most countable and as $m$ is proper, each critical level has only finitely many critical points. In fact, it may be assumed that there is only one critical point on each critical level and that $P_{0}=m^{-1}(0)$ is a critical point of index 0 . Now $m$ has an associated gradient vector field $V$. We will say that a trajectory of $V$ is critical if its closure contains two critical points, i.e., it runs between two critical points. By altering $V$ between critical levels (if necessary), it can be arranged that all noncriticial trajectories of $V$ do not terminate in finite time, i.e., they take "infinite time to reach $\infty$ ". Note that any noncritical trajectory starting at a noncritical level $m^{-1}(r), r>0$, has a parametrization $\gamma:[r, \infty) \rightarrow M$ such that $m \gamma(x)=x \forall x \in[r, \infty)$. From this, it can be seen that noncritical trajectories give rise to proper embeddings.

We now construct $g$. Let $\theta: B \rightarrow M$ be a coordinate chart around $P_{0}$ where $B$ is the open unit 3-ball $\subset \mathbf{R}^{3}$. We may assume that in coordinates $(x, y, z)$ with respect to $\theta, V(x, y, z)=(x, y, z)$. Any diameter of $B$ will therefore determine a pair of trajectories emanating from $P_{0}$. As the set of critical trajectories is at most countable, there is a diameter of $B$ determining two noncritical trajectories. The closure of these two trajectories defines a smooth submanifold $G \subset M$, i.e., we have a smooth, proper embedding $g: \mathbf{R} \rightarrow M$ such that $g(\mathbf{R})=G, g(0)=P_{0}$ and $g^{\prime}$ agrees with $V$ (up to sign) outside $\theta(B)$.

We now use the flow of $V$ to get a smooth, proper isotopy connecting an arbitrary smooth proper embedding, $f_{0}: \mathbf{R} \rightarrow M$, to $g$. First isotope $f_{0} \mid[-1,1]$ to $g \mid[-1,1]$ and then extend by the isotopy extension theorem to get a smooth, proper embedding $f_{1}: \mathbf{R} \rightarrow M$. Then isotope $f_{1}$ off all critical points (except $P_{0}$ ) by 


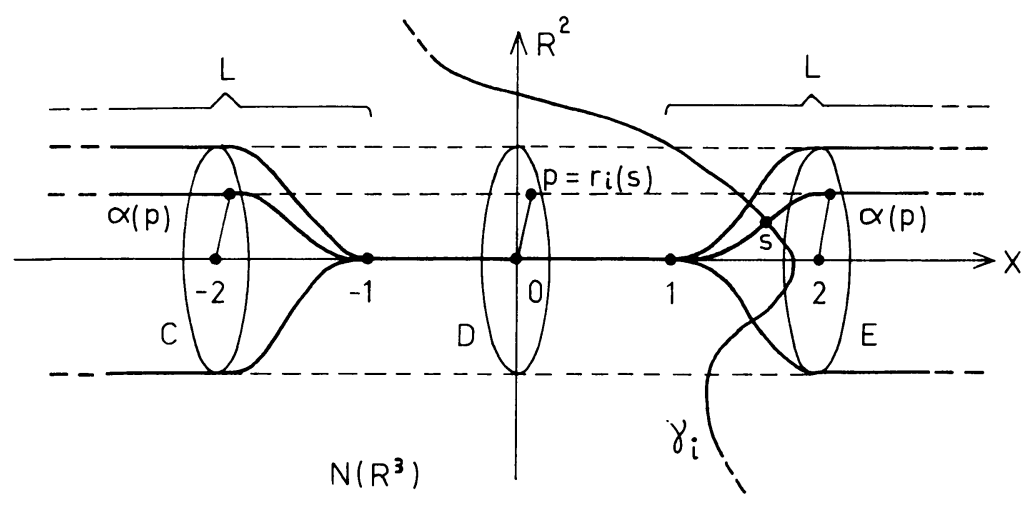

FIGURE 6

deforming it locally in small neighbourhoods around each critical point to obtain another smooth, proper embedding $f_{2}: \mathbf{R} \rightarrow M$ such that $f_{2}|[-1,1]=g|[-1,1]$. We are almost ready to comb $f_{2}$ out along $g$ using the combing out vector field $V$, but we must arrange that $f_{2}$ misses all critical trajectories beforehand. So let $N: \mathbf{R} \times \mathbf{R}^{2} \rightarrow M$ be a product neighbourhood of $f_{2}$ which misses all critical points except $P_{0}$ and is such that $N(r, 0)=f_{2}(r) \forall r \in \mathbf{R}$. Denote by $(x,(y, z))$ coordinates with respect to $N$. Now set up a "pinched pencil" of curves in $N\left(\mathbf{R}^{3}\right)$ parallel to the $(x, 0,0)$ axis as in Figure 6. $C, D$ and $E$ are open 2-discs, radius $=1$, perpendicular to the $(x, 0,0)$ axis at $x=-2,0,2$ respectively. Each $p \in D$ determines a smooth curve $\alpha(p): \mathbf{R} \rightarrow N\left(\mathbf{R}^{3}\right)$ given, in coordinates with respect to $N$, by

$$
\begin{aligned}
\alpha(p)(r) & =(r, p), & & |r| \geq 2, \\
& =(r, 0), & & |r| \leq 1,
\end{aligned}
$$

and for $1 \leq|r| \leq 2$, join $( \pm 2, p)$ smoothly to $( \pm 1,0)$ by a family of smooth curves depending smoothly on $p$ and such that each resulting $\alpha(p)$ is smooth. Thus we have a smooth map $\alpha: D \times \mathbf{R} \rightarrow N\left(\mathbf{R}^{3}\right)$ where each $\alpha(p, \cdot)=\alpha(p)$ runs parallel to $f_{2}(r)$ in $N\left(\mathbf{R}^{3}\right),|r| \geq 2$, and agrees with $g$ on $[-1,1]$. Let $L \subset N\left(\mathbf{R}^{3}\right)$ be the smooth submanifold $\alpha(D,\{|x|>1\})$ and let the critical trajectories of $V$ be $\gamma_{0}, \gamma_{1}, \gamma_{2}, \ldots$ Then for $i=0,1,2, \ldots$, define $r_{i}: \gamma_{i} \cap L \rightarrow D$ to be projection first along the "pencil curves", $\alpha(p)$, to $C$ or $E$ (depending on which component of $L$ that $s \in \gamma_{i} \cap L$ lies in) and then to $D$ along straight lines parallel to the $(x, 0,0)$ axis. So $r_{i}(s)=p \Rightarrow s \in \alpha(p)(\mathbf{R})$. By Sard's theorem, each $R_{i}=r_{i}\left(\gamma_{i} \cap L\right)$ has measure 0 in $D$ and hence so does $\bigcup_{i} R_{i}$. We can therefore find $p^{\prime} \in D-\bigcup_{i} R^{i}$ so that $f=\alpha\left(p^{\prime}\right): \mathbf{R} \rightarrow M$ is a smooth, proper embedding, smoothly isotopic to $f_{2}$. It avoids all critical points and critical trajectories and is such that $f \mid[-1,1]=$ $g \mid[-1,1]$. Notice that this method of separating $f_{2}$ from critical trajectories will not work, in general, in the presence of critical points of index 2. Notice also that each isotopy in the chain $f_{0} \rightarrow f_{1} \rightarrow f_{2} \rightarrow f$ is proper.

Consider the map $F: \mathbf{R} \times[0, \infty) \rightarrow M$ given by $F_{\mu}(t)=\chi_{\mu}\left[f\left(h_{\mu}(t)\right)\right]$ where $\mu \in[0, \infty), t \in \mathbf{R}, \chi: M \times[0, \infty) \rightarrow M$ is the flow of $V, \chi_{\mu}$ is the $\mu$ th stage and $h_{\mu}=g^{-1} \chi_{\mu}^{-1} g: \mathbf{R} \rightarrow \mathbf{R}$. To understand $F$, we will first examine the behaviour of $\chi_{\mu}[f(\mathbf{R})]$ as $\mu \rightarrow \infty$. Let $K \subset M$ be compact and let $K \subset M(a)=m^{-1}([0, a])$ for some $a>0$. Also let $A \subset M(a)$ be a small, closed 3-ball around $P_{0}$ such that 


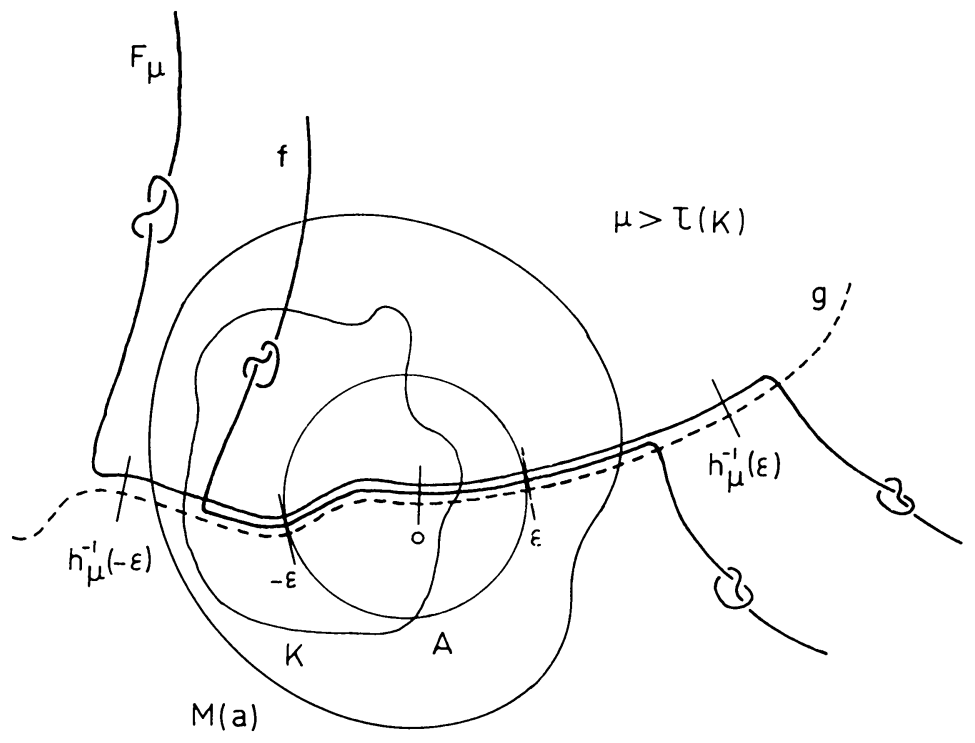

FIGURE 7

$A \cap f(\mathbf{R})=f([-\varepsilon, \varepsilon])$ for some $0<\varepsilon<1$. Then as the set $S=f(\{|r| \geq \varepsilon\}) \cap M(a)$ is compact, there is a stage $=\tau(K)>0$ such that

$$
\forall \mu>\tau(K), \quad \chi_{\mu}(S) \cap M(a)=\varnothing .
$$

In fact, since trajectories do not return to a level surface like $m^{-1}(a)$ having once left, then

$$
\chi_{\mu}[f(\{|r| \geq \varepsilon\})] \cap M(a)=\varnothing \quad \forall \mu>\tau(K) .
$$

So in a manner similar to the previous proposition, under the map $\chi_{\mu}$ and as $\mu \rightarrow \infty$, the 3-ball $A$ expands without bound along noncritical trajectories and combs out $f$ along $g$, leaving $g$ inside it. The function $h_{\mu}$ ensures that the correct parametrization is obtained. That is, at stage $0, F_{0}|[-\varepsilon, \varepsilon]=f|[-\varepsilon, \varepsilon]=g \mid[-\varepsilon, \varepsilon]$, and at stage $\mu$,

$$
\begin{aligned}
F_{\mu} h_{\mu}^{-1} \mid[-\varepsilon, \varepsilon] & =\chi_{\mu} f\left|[-\varepsilon, \varepsilon]=\chi_{\mu} g\right|[-\varepsilon, \varepsilon] \\
& =g g^{-1} \chi_{\mu} g\left|[-\varepsilon, \varepsilon]=g h_{\mu}^{-1}\right|[-\varepsilon, \varepsilon] .
\end{aligned}
$$

So $F_{\mu}$ and $g$ agree on the interval $h_{\mu}^{-1}([-\varepsilon, \varepsilon])$, and as $\mu \rightarrow \infty, h_{\mu}^{-1}([-\varepsilon, \varepsilon]) \rightarrow \mathbf{R}$.

Finally, the required isotopy concerning $f$ and $g, H: \mathbf{R} \times[0,1] \rightarrow M$, can be defined using $F$ and a diffeomorphism $\lambda:[0,1) \rightarrow[0, \infty)$, i.e., $H_{\rho}=F_{\lambda(\rho)}$ for $\rho \in[0,1)$ and $H_{1}=g . H$ is clearly smooth on $\mathbf{R} \times[0,1)$ and is smooth at $\mathbf{R} \times\{1\}$ since $H_{\rho}$ and $g$ agree on $h_{\lambda(\rho)}^{-1}([-\varepsilon, \varepsilon])$ which $\rightarrow \mathbf{R}$ as $\rho \rightarrow 1$.

To show $H$ is proper, it suffices to show, as in the previous proposition, that wd $H^{-1}(K)<\infty$ for our arbitrary $K \subset M$. So let $\omega=\lambda^{-1}(\tau(K)), \tau(K)$ as in the preceding discussion, and then

$$
\operatorname{wd} H^{-1}(K)=\mathrm{wd}\left[\left(\bigcup_{0 \leq \rho \leq \omega} H_{\rho}^{-1}(K) \times\{\rho\}\right) \cup\left(\bigcup_{\omega \leq \rho \leq 1} H_{\rho}^{-1}(K) \times\{\rho\}\right)\right] .
$$


But by the definition of $\tau(K)$ and $F, F_{\mu}^{-1}(K)=g^{-1}(K) \forall \mu \geq \tau(K)$. Thus

$$
\operatorname{wd}\left[\bigcup_{\omega \leq \rho \leq 1} H_{\rho}^{-1}(K) \times\{\rho\}\right]=\operatorname{wd}\left[g^{-1}(K) \times[\omega, 1]\right]<\infty \quad \text { (since } g \text { is proper). }
$$

Also note that since $K \subset M(a)$, then under the "backward flow", $\chi_{\mu}^{-1}(K) \subset M(a)$ $\forall \mu \geq 0$. So,

$$
\begin{aligned}
\mathrm{wd}\left[\bigcup_{0 \leq \rho \leq \omega} H_{\rho}^{-1}(K) \times\{\rho\}\right] & =\mathrm{wd}\left[\bigcup_{0 \leq \mu \leq \tau(K)} h_{\mu}^{-1} f^{-1} \chi_{\mu}^{-1}(K) \times\{\mu\}\right] \\
& \leq \mathrm{wd}\left[\bigcup_{0 \leq \mu \leq \tau(K)} h_{\mu}^{-1} f^{-1}(M(a)) \times\{\mu\}\right] \\
& \leq \mathrm{wd}\left[\bigcup_{0 \leq \mu \leq \tau(K)} h_{\mu}^{-1}([-t, t]) \times\{\mu\}\right]
\end{aligned}
$$

for some large constant $t \in \mathbf{R}$ (since $f$ is proper). But

$$
\forall 0 \leq \mu \leq \mu^{\prime}, \quad h_{\mu}^{-1}([-t, t]) \subset h_{\mu^{\prime}}^{-1}([-t, t]),
$$

and $\forall \mu \geq 0, h_{\mu}^{-1}([-t, t])$ is compact (since $h_{\mu}^{-1}$ is continuous). So,

$$
\mathrm{wd}\left[\bigcup_{0 \leq \mu \leq \tau(K)} h_{\mu}^{-1}([-t, t]) \times\{\mu\}\right]<\mathrm{wd}\left[h_{\tau(K)}^{-1}([-t, t]) \times[0, \tau(K)]\right]<\infty .
$$

Hence wd $H^{-1}(K)<\infty$.

The manifolds that are being considered in the above theorem are really just open, solid tori and Klein bottles with countably many holes. With such a classification, the proposition may be proved in an analogous way by using the radial vector field associated with a proper deformation retract to a spine.

Theorem A can be generalised as follows.

THEOREM B. Let $M$ be an open, connected, smooth, $r$-dimensional manifold, $r \geq 2$, which admits a smooth, proper Morse function $m: M \rightarrow \mathbf{R}$ such that

(i) $m(M)=[0, \infty)$,

(ii) the critical points of $m$ are of index $\leq r-p-1$, where $0<p<r$.

Then up to smooth, proper isotopy, there is a unique, smooth, proper embedding $g: \mathbf{R}^{p} \rightarrow M$.

COROLLARY. In the above, if $M$ is a 4-manifold and $m$ has critical points all of index $\leq 1$, then up to smooth, proper isotopy, there is a unique, smooth, proper embedding $g: \mathbf{R}^{2} \rightarrow M$.

The proof of Theorem B follows that of Theorem A, but more care must be taken when constructing the initial embedding $g$. In Theorem A, we found an embedded 3-ball around $P_{0}$, with boundary $\Sigma^{3}$, such that all trajectories passing through $\Sigma^{3}$ were radial from $P_{0}$. We then used an embedded 0 -sphere, $\Sigma^{0} \subset \Sigma^{3}$, to specify two noncritical trajectories emanating from $P_{0}$ and passing through $\Sigma^{0}$. These then 
gave rise to $g$. In Theorem B, again using Sard's theorem, we actually find an unknotted $\Sigma^{p-1} \subset \Sigma^{r-1}$ around $P_{0}$ such that all trajectories passing through it are noncritical. This cone of trajectories is then (after smoothing the vertex) used to define $g$.

3. Concluding remarks. 1. Let $M$ be an open 3-manifold and $f: \mathbf{R} \rightarrow M$ a proper knot. Suppose $g: B^{3} \rightarrow M$ is a smooth embedding of a 3-ball such that $g\left(B^{3}\right) \cap f(\mathbf{R})$ is an embedded arc $A$ which meets $g\left(\partial B^{3}\right)$ transversely in two points. Then, by setting up an appropriate vector field in a tubular neighbourhood of $f$ and in $g\left(B^{3}\right)$, the arc $A$ can be combed to infinity by a smooth, proper isotopy. In this way, $f$ is equivalent to a proper knot in $M$ which is trivial inside the 3-ball $g\left(B^{3}\right)$. This construction suggests the following conjecture.

CONJECTURE. A proper knot $f: \mathbf{R}^{1} \rightarrow M$ is properly isotopic to a proper knot $f_{0}: \mathbf{R}^{1} \rightarrow M$ which is "locally trivial" in the following sense: Let $g: B^{3} \rightarrow M$ be any smooth embedding of a 3-ball such that $g\left(B^{3}\right) \cap f_{0}(\mathbf{R})$ is an embedded arc $A$ which meets $g\left(\partial B^{3}\right)$ transversely in two points $\{a, b\}$. Then for all such $g, A$ is unknotted in $g\left(B^{3}\right) \operatorname{rel}\{a, b\}$.

The conjecture implies that proper knot theory reduces to the study of locally trivial proper knots (e.g., the proper knot $K_{m}$ in Figure 1 is locally trivial).

2. Let $M$ be a compact 3-manifold, $\partial M \neq \varnothing$, which is a handlebody consisting of 0 - and 1-handles only. Let $K$ be a proper knot in $\operatorname{int}(M)$. The combing process of Theorem A shows also that $K$ can be engulfed from $\partial M$ in $M$. This therefore suggests a different approach to engulfing in connected 3-manifolds: Instead of trying to engulf a knot from a given 3 -ball, one tries to comb the knot into the 3 -ball along a suitable vector field. This approach to engulfing will be explained in a forthcoming paper by the second-named author.

\section{REFERENCES}

1. G. Bourgeois and D. Spring, Groupes d'homotopie de l'espace des plongements propres d'une nvariété ouverte dans une m-variété ouverte, C. R. Acad. Sci. Paris Sér. A 286 (1978), 771-773.

2. R. H. Fox, A remarkable simple closed curve, Ann. of Math. (2) 50 (1949), 264-265.

3. J. Milnor, Lectures on the $h$-cobordism theorem, Princeton Univ. Press, 1965.

4. D. Rolfsen, Knots and links, Publish or Perish, 1976.

5. D. Spring, Proper homotopy and immersion theory, Topology 11 (1971), 295-305.

Department of Mathematics, University of toronto, Toronto, Ontario M5S 1A1, CANADA

Department of Mathematics, Glendon College, York University, 2275 Bayview avenue, Toronto, Ontario M4N 3M6, Canada 